\title{
Dynamic Property Analysis of At-Ground Structure Under Moving Maglev Train
}

\author{
Dangxiong Wang ${ }^{1} \mathbb{D} \cdot$ Xiaozhen $_{\mathrm{Li}^{1}} \cdot \mathrm{Jie}_{\text {Geng }}{ }^{1} \cdot$ Yanlong Ge$^{1}$
}

Received: 2 August 2017/Revised: 2 September 2017/Accepted: 4 September 2017/Published online: 18 September 2017

(c) The Author(s) 2017. This article is an open access publication

\begin{abstract}
In order to study the vertical coupling vibration of low-medium-speed Maglev train and at-ground structure system, the vertical coupling vibration model of lowmedium-speed Maglev train-bridge system is established firstly based on the co-simulation of SIMPACK and ANSYS. The method of co-simulation is verified through the test experiments on a 20-m simply supported beam dynamic load experiment test line. Later, the vertical coupling vibration dynamics simulation model of lowmedium-speed Maglev train and at-ground structure system is built and the dynamic simulation analysis is performed. According to the analysis, the vibration frequency of the atground structure is relatively high and the first vertical vibration frequency is $32.9 \mathrm{~Hz}$; the vertical displacement and acceleration of the frame's center of the at-ground structure are bigger than the bottom's center; the vibration of the frame is the high-frequency vibration (comparing with the bottom), and the acceleration of the frame's center is obviously greater than the bottom's between 50 and $100 \mathrm{~Hz}$.
\end{abstract}

Keywords Low-medium-speed Maglev train - At-ground structure Coupling vibration · High-frequency vibration . Co-simulation

Dangxiong Wang

Dangxiong333@163.com

1 Department of Bridge Engineering, School of Civil Engineering, Southwest Jiaotong University, Chengdu 610031, China

Editor: Eryu Zhu

\section{Introduction}

Nowadays, with the rapid growth of China's economic and urban population, a fast, economic land occupation, environment protection way of transport is eagerly needed. Maglev transport has been born with many advantages, including well climbing capacity, small turning radius, low noise, low pollution, and good environmental compatibility. As a representative of maglev transportation, lowmedium-speed maglev transportation is particularly suitable for medium-short-distance transport in cities and has good development in the future urban transportation [1].

The low-medium-speed maglev line consists of bridges and subgrade structure. Because the Maglev train embraces the rail, in subgrade section, the maglev guideway has to be propped up by a special structure and ensure the Maglev train having enough space to embracing the rail in vertical. The structure is called at-ground structure [2]. The atground structure is a concrete beam which is placed on the processing subgrade in advance and supported by uniform elastic subgrade, forming the combination system of Maglev train-at-ground structure subgrade. Low-mediumspeed Maglev train stabilizes near the rated suspension gap through adjusting the active control electromagnetic force and keeps running smoothly. Therefore, both the bridge and at-ground structure will produce deformation under the action of Maglev train and affect the suspension gap, then affecting the smooth running of the Maglev train. Lots of scholars are all committed to Maglev train-bridge system coupling vibration: Ref. [3] analyzed the bridge dynamic responses when the high-speed Maglev train runs on the bridge at the resonance speed; Ref. [4] considering the PI control and establishing two degrees of freedom Maglev train-simply supported beam (simplified as Euler-Bernoulli beam) vertical coupling vibration model, discussed the 
resonance effect of coupling model and the influences of the suspension control parameters on the coupling vibration; Ref. [5] building 10 degrees of freedom TR06 Maglev train model and simplifying the suspension control as linear spring-damping system, discussed the Maglev trainbridge vertical coupling vibration under random irregularity excitation; Refs. [6-9] establishing Maglev train-bridge vertical coupling vibration model, studied the effect of bridge stiffness, material and structure form to the dynamic responses of Maglev train-bridge system; Refs. [10, 11] analyzed dynamic characters of suspension control system and bridge and also studied the changing rules of suspension gaps at different spans and bridge stiffness; Refs. [12] considering 12 degrees of freedom Maglev train model and PD control, discussed the impact effect of Maglev train to bridge; Refs. [13, 14] analyzed the influence of wind load on the coupling vibration of Maglev train-bridge system.

The at-ground structure is supported by the uniform elastic subgrade. Under the train load, the stress mechanism is obviously different from the bridge. Under the long time operation, some uncontrollable factors will affect the Maglev train-at-ground structure vertical coupling vibration, including the supported stiffness change of subgrade, uneven settlement and the supported void. The at-ground structure is generally used frame structure, and ordinary Euler-Bernoulli beam model cannot predict the dynamic performance accurately. So, it is necessary to research the Maglev train-at-ground structure system vertical coupling vibration and the related articles is little. On the basis of predecessors' research, this paper considered the PID active suspension control and used the delicate FEM model of bridge and at-ground structure based on co-simulation of SIMPACK and ANSYS. Firstly, the Maglev train-bridge vertical system coupling vibration model was validated based on one 20-m simply supported beam load test of a low-medium-speed maglev test line. Then, the Maglev train-at-ground structure vertical system coupling vibration model was established to be analyzed.

\section{Theoretical Model}

\subsection{The Model of Maglev Train}

In this paper, the five modules low-medium-speed Maglev train was considered. Each car is supported by five pairs of suspension frames. The vehicle and suspension frame are connected through air spring, slide, etc. Each suspension frame has four independent suspension electromagnets, which produced levitation force and act on the suspension frame and bridge to achieve the maglev car suspension steady. The vehicle has 40 suspension electromagnets totally, and the neighboring electromagnets longitudinal distance is $0.7 \mathrm{~m}$. Figure 1 shows the Maglev train suspension.

Car body and suspension frame are both considered six degrees of freedom. The air spring is a treated springdamping element. The vehicle's degree of freedom is shown in Table 1, and the total number is 86 . According to d'Alembent, the movement differential equations of car body and suspension frames are established [1]. Table 2 shows the main calculation parameters of Maglev train.

\subsection{Suspension Control Model}

The relationship among electromagnetic force, suspension gap and current is [15]:

$F(c, i(t))=\frac{\mu_{0} A n^{2}}{4}\left[\frac{i_{0}+\Delta i(t)}{c_{0}+\Delta c(t)}\right]^{2}$

This paper considers PID control based on displacement-speed-acceleration feedback. Because the speed signal cannot be measured directly, it should be reconstructed by displacement and acceleration signals in the state observer. The state of construction is as follows:

$\Delta \dot{\hat{c}}(t)=\Delta \dot{\hat{z}}(t)+2 \xi_{0} \omega_{0}[\Delta c(t)-\Delta \hat{c}(t)]$

$\Delta \ddot{\hat{z}}(t)=\Delta \ddot{z}(t)+\omega_{0}^{2}[\Delta c(t)-\Delta \hat{c}(t)]$

After using state reconstruction, feedback current can be expressed as the function of displacement speed, acceleration feedback signal, as follows:

$\Delta i(t)=K_{p} \Delta c(t)+K_{v} \Delta \dot{\hat{c}}(t)+K_{a} \Delta \ddot{z}(t)$

So, the electromagnetic levitation force of control point is shown as:

$F(c, i(t))=\frac{\mu_{0} A n^{2}}{4}\left[\frac{i_{0}+K_{p} \Delta c(t)+K_{v} \Delta \dot{\hat{z}}(t)+K_{a} \Delta \ddot{z}(t)}{c_{0}+\Delta c(t)}\right]^{2}$

The levitation force is applied to the electromagnet, and the active suspension control of Maglev train can be realized.

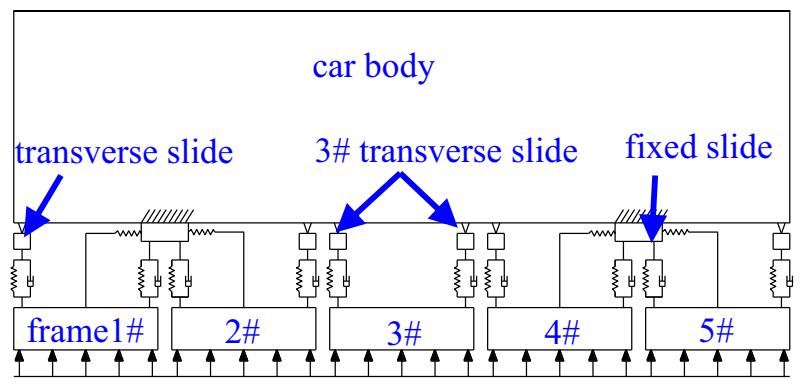

Fig. 1 Suspension of five modules $L \_M$-speed Maglev train 
Table 1 Dofs of the vehicle model

\begin{tabular}{lccccccc}
\hline Component & $x$ & $y$ & $z$ & $\alpha$ & $\beta$ & $\gamma$ & Remark \\
\hline Suspension frame & $x_{f i}$ & $y_{f i}$ & $z_{f i}$ & $\alpha_{f i}$ & $\beta_{f i}$ & $\gamma_{f i}$ & $i=1-10$ \\
3\# sliding table & - & $y_{s m i}$ & - & - & - & - & $i=1-4$ \\
Other sliding tables & $x_{s i}$ & $y_{s i}$ & - & - & - & - & $i=1-8$ \\
Vehicle & $x_{c i}$ & $y_{c i}$ & $z_{c i}$ & $\alpha_{c i}$ & $\beta_{c i}$ & $\gamma_{c i}$ & $i=1$ \\
\hline
\end{tabular}

Table 2 Calculation parameters of the Maglev train (30t)

\begin{tabular}{llll}
\hline Sign & Name & Unit & Parameters \\
\hline$M_{c}$ & Vehicle mass & $\mathrm{kg}$ & $18.77 \times 10^{3}$ \\
$M_{f}$ & Frame mass & $\mathrm{kg}$ & 1000 \\
$I_{c x}$ & Vehicle around $x$-axis & $\mathrm{kg} \mathrm{m}^{2}$ & $2.21 \times 10^{3}$ \\
$I_{c y}$ & Vehicle around $y$-axis & $\mathrm{kg} \mathrm{m}^{2}$ & $3.85 \times 10^{5}$ \\
$I_{c z}$ & Vehicle around $z$-axis & $\mathrm{kg} \mathrm{m}^{2}$ & $3.88 \times 10^{5}$ \\
$I_{f x}$ & Frame around $x$-axis & $\mathrm{kg} \mathrm{m}^{2}$ & 460 \\
$I_{f y}$ & Frame around $y$-axis & $\mathrm{kg} \mathrm{m}^{2}$ & 1150 \\
$I_{f z}$ & Frame around $z$-axis & $\mathrm{kg} \mathrm{m}$ & 1200 \\
$K_{s z}$ & Air spring stiffness & $\mathrm{kN} / \mathrm{m}$ & 80 \\
$C_{s z}$ & Air spring damping & $\mathrm{kN} / \mathrm{s} / \mathrm{m}$ & 5 \\
\hline
\end{tabular}

\subsection{The Model of At-Ground Structure}

The at-ground structure is a concrete beam which is placed on the processing subgrade in advance and supported by uniform elastic subgrade. It consists of five spans continuous rigid bridge (on top) and bottom. Subgrade consists of graded crushed stone and $\mathrm{AB}$ packing and the function of subgrade likes stiffness. As shown in Fig. 2, the subgrade is simplified uniform spring for theoretical model. Figure 3 shows the FE model of at-ground structure.

Because of using the modal superposition, SIMPACK eventually recognizes the modal information of at-ground structure. ANSYS has the powerful flexible body modal analysis function and can build any exquisite flexible structure model. So, for any structure (including bridge), this co-simulation method can be adopted and the theory is the same.

\section{Model Validation}

This co-simulation method was verified based on the 20-m simply supported beam dynamic load experiment of a test line. Using the same theory, the Maglev train-bridge vertical system coupling vibration model was established. Figure 4 shows the middle span section of the simply supported beam, using C50 concrete.
Using three Maglev train marshalings, the weight is $25 t+30 t+30 t$ and the velocity of train is $80 \mathrm{~km} / \mathrm{h}$. The track irregularity spectrum recommended by Ref. [16] is adopted. Figure 5 shows the comparison of the simulation and measurement time-history curves of vertical dynamic deformation and acceleration in middle span of the simply supported beam. Figure 6 shows the comparison of the simulation and measurement frequency-history curves of acceleration in middle span of the simply supported beam.

According to the comparison, in both time-history and frequency-history, the simulation values are fit with the measured values. That is to say, the Maglev train-bridge vertical system coupling vibration model based on cosimulation method is reliable. Because of the same simulation theory, the Maglev train-at-ground structure vertical system coupling vibration model is also reliable.

\section{Analysis of Maglev Train-At-Ground Structure Vertical System Coupling Vibration}

\subsection{Natural Frequency of At-Ground Structure}

The at-ground structure FE model was established in ANSYS using solid95-type element and C40 concrete. The combin14-type element was used to simulate the uniform stiffness, and the stiffness value is $200 \mathrm{MPa} / \mathrm{m}$. The section size is shown in Fig. 2.

Table 3 shows the nature frequency of the at-ground structure within $100 \mathrm{~Hz}$. Figure 7 shows the typical vibration modes. As shown in Table 3 and Fig. 7, the nature frequencies of the at-ground structure are obviously high. There are only seven modes within $100 \mathrm{~Hz}$, and the first vertical nature frequency is $32.9 \mathrm{~Hz}$, and before the $32.9 \mathrm{~Hz}$, there have no vertical vibration modes. The reason is the at-ground structure is supported by the strong uniform elastic subgrade and its stiffness is big. Before 80 $\mathrm{Hz}$, the vibration is mainly the overall vertical modes. In the second overall vertical modes, there also has the frame's local bend vibration.

\subsection{Dynamic Response Analysis of the At-Ground Structure}

Using three Maglev train marshalings, the weight is $25 t+30 t+30 t$ and the velocity of train is $80 \mathrm{~km} / \mathrm{h}$.

Figure 8 shows the vertical dynamic deformation in frame's center (as shown in Fig. 2, point B) and bottom's center (as shown in Fig. 2, point A) of at-ground structure. As shown in Fig. 8, the dynamic deformation time-history curves frame's center and bottom's center both have three peaks because of the three Maglev train marshalings. The maximum value is $0.466 \mathrm{~mm}$ (frame's center) and 
Fig. 2 At-ground structure simplified model (unit: $\mathrm{mm}$ ). a Front view. b Side view

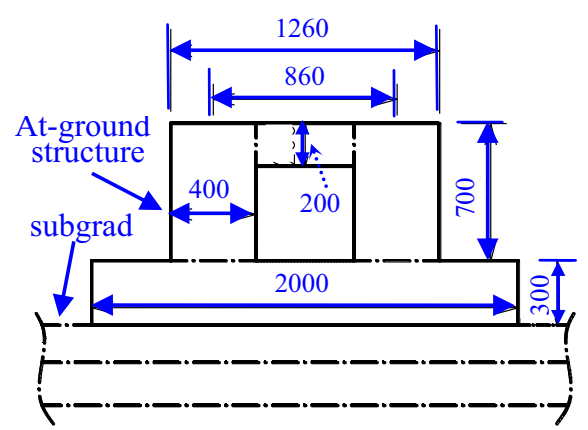

(a) Front view

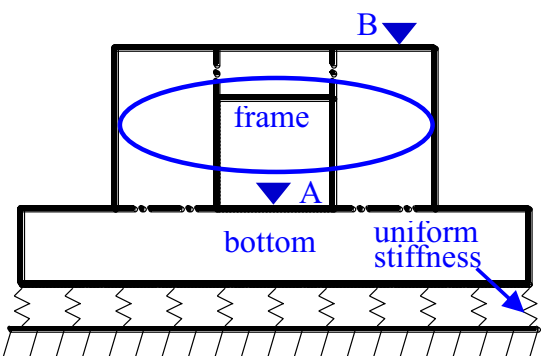

$13200 / 2$

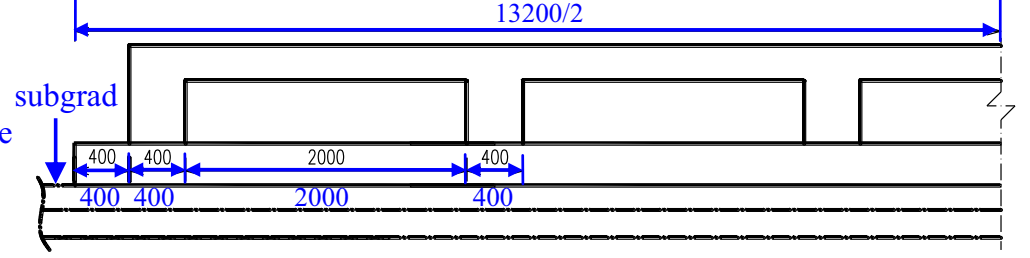

$\mathrm{B}$

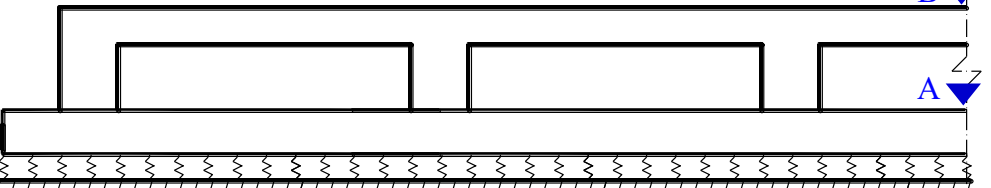

(b) Side view

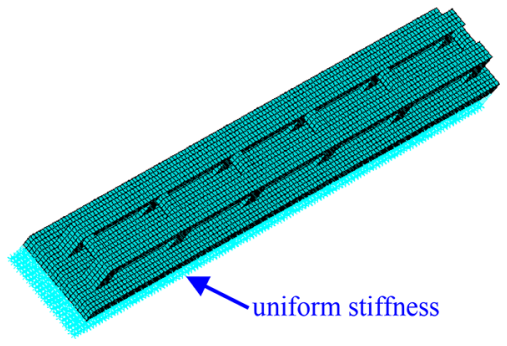

Fig. 3 At-ground structure FE model

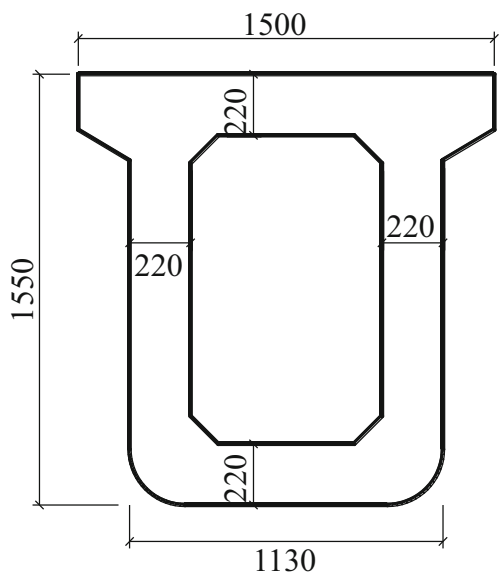

Fig. 4 Cross section of 20-m simply supported beam (unit: $\mathrm{mm}$ )
$0.378 \mathrm{~mm}$ (bottom's center), respectively. Because the total dynamic deformation of frame's center is composed of the total bottom's center dynamic deformation and the local frame's center dynamic deformation, the dynamic deformation of frame's center is bigger than the bottom's center dynamic deformation.

Figure 9 shows the vertical acceleration in frame's center and bottom's center of at-ground structure. As shown in Fig. 9, the peak values of vertical acceleration in frame's center and bottom's center are 6.017 and $1.428 \mathrm{~m} /$ $\mathrm{s}^{2}$. The value of frame's center is much larger than the value of bottom's center. In order to analysis the vibration characteristics of frame and bottom's center in-depth, Fig. 10 shows the acceleration frequency-history curves of frame's center and bottom's center. As shown in Fig. 10, there are obviously acceleration peaks of frame's center and bottom's center within $100 \mathrm{~Hz}$ and the frequency is frame's center: 31.2, 62.5, 87.8, 95.7 Hz; bottom's center: 31.2, 62.5, 95.7 Hz. Before the $50 \mathrm{~Hz}$, the acceleration of frame's center is little different with bottom's center acceleration and after the $50 \mathrm{~Hz}$, the acceleration of frame's center is much bigger than bottom's center acceleration.

In order to analyze the vibration, due to the natural vibration analysis of at-ground structure, the first and second frequency of vertical overall bend vibration is 32.9 and $62.4 \mathrm{~Hz}$ separately and at $88.2,91.4,97.2 \mathrm{~Hz}$, there is 


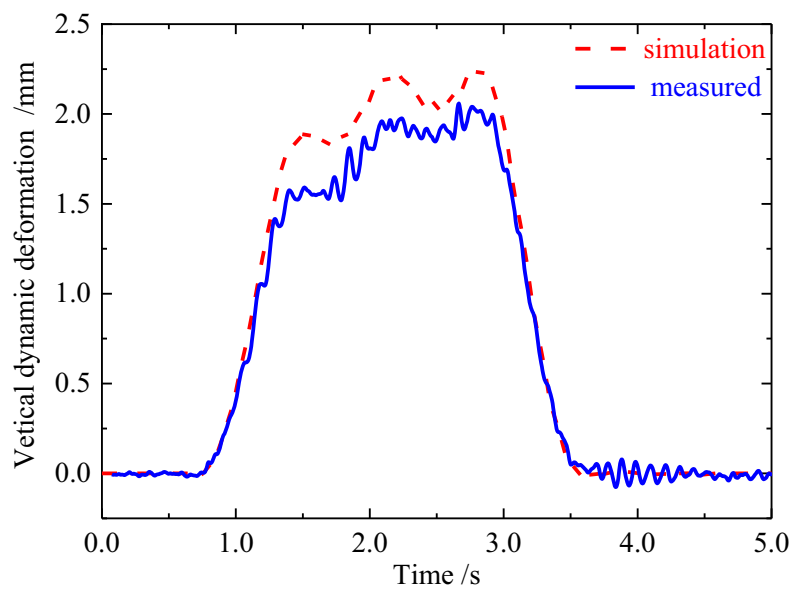

(a) Dynamic deformation

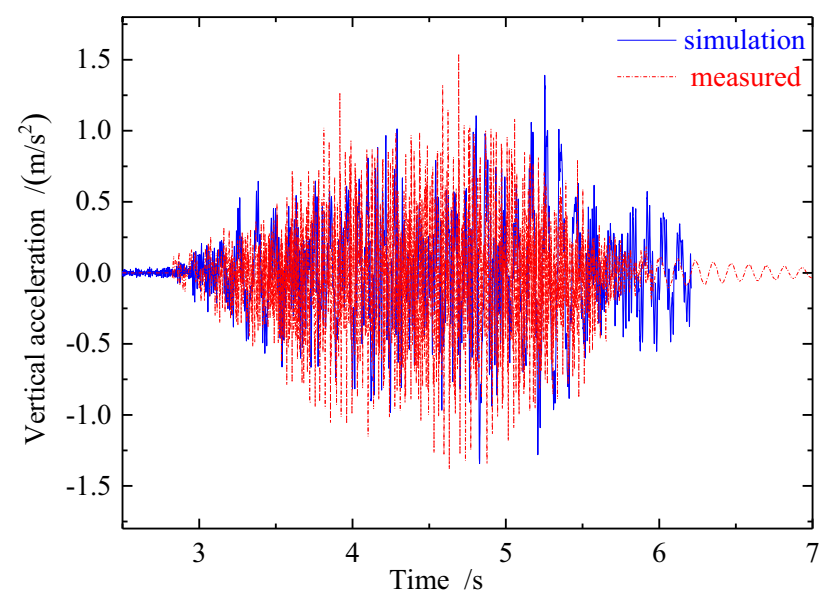

(b) acceleration

Fig. 5 Comparison of the simulation and measurement time-history curves. a Dynamic deformation. b Acceleration

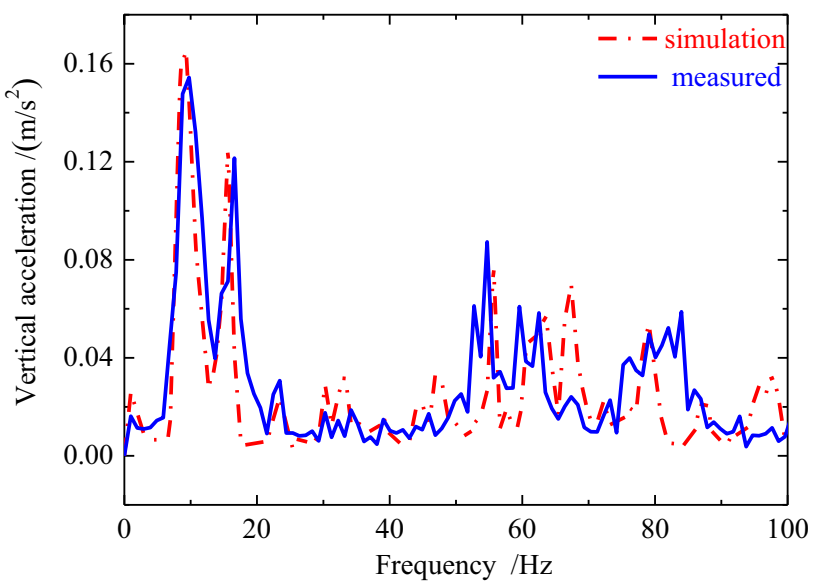

Fig. 6 Comparison of the simulation and measurement frequencyhistory curves

frame's local vertical vibration. So, the vibration peaks of frame's center and bottom's center are existed at this
Table 3 The modal and nature frequency of at-ground structure

\begin{tabular}{ll}
\hline Frequency & Modal \\
\hline 32.9 & 1st symmetric overall vibration \\
45.6 & 1st antisymmetric overall vibration \\
62.4 & 2nd symmetric overall vibration \\
77.5 & 2nd antisymmetric overall vibration \\
88.2 & Frame local vibration \\
91.4 & Frame local vibration \\
97.2 & Frame local vibration \\
\hline
\end{tabular}

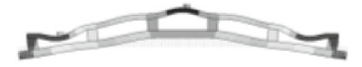

1th symmetric overall

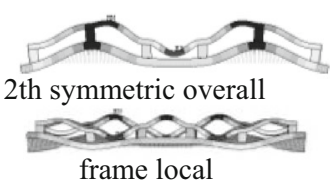

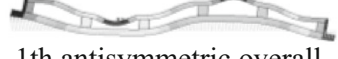

1 th antisymmetric overall

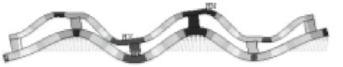

2th antisymmetric overall

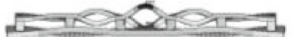

frame local

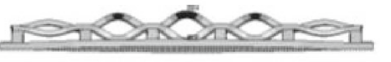

frame local

Fig. 7 The typical modal of at-ground structure

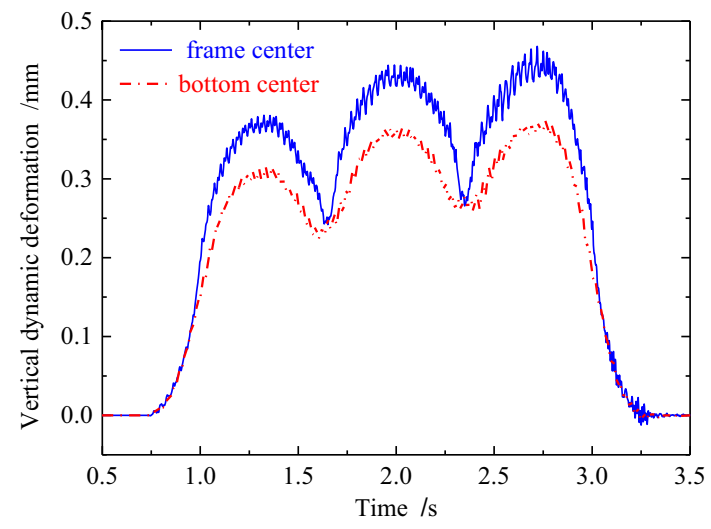

Fig. 8 The vertical dynamic displacement time-history curves of atground structure

frequency. From the second vertical bend modal $(62.4 \mathrm{~Hz})$, the frame's local vertical vibration modal appeared. So, before about $50 \mathrm{~Hz}$, because of the overall vertical vibration of at-ground structure, the acceleration values of frame's center and bottom's center are nearly equal and between 50 and $100 \mathrm{~Hz}$, and the acceleration of frame's center is much bigger than bottom's center acceleration based on the frame's local vertical vibration. Therefore, the frame's vertical vibration is high frequency (compared with the bottom's vibration). 


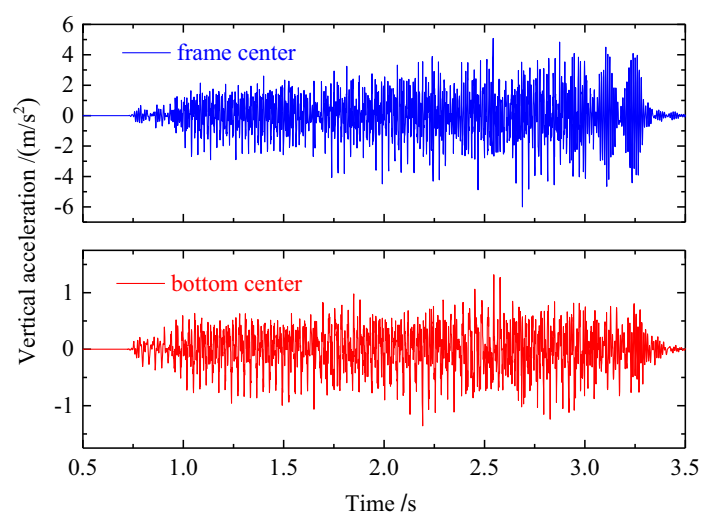

Fig. 9 The vertical acceleration time-history curves of at-ground structure

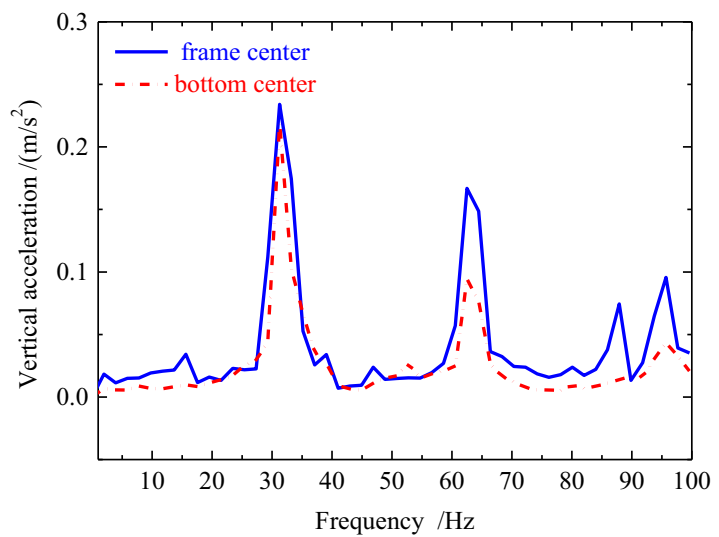

Fig. 10 The vertical acceleration frequency-history curves of atground structure

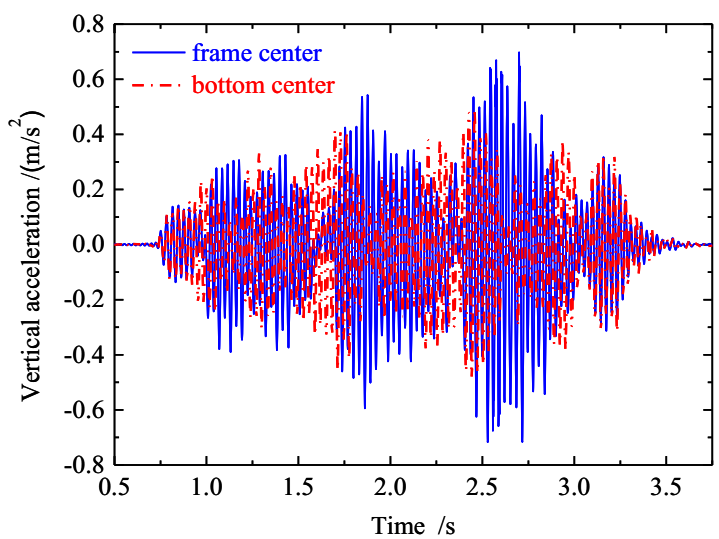

Fig. 11 The vertical acceleration of at-ground structure $(50-\mathrm{Hz}$ lowpass filter)

Figure 11 shows the vertical acceleration time-history curves of frame's center and bottom's center $(50-\mathrm{Hz}$ lowpass filter).

As shown in Fig. 11, comparing with the unfiltered curves, the $50-\mathrm{Hz}$ low-pass filter acceleration curves of frame's center are little different with bottom's center acceleration curves and both the curves $(50-\mathrm{Hz}$ low-pass filter) have obviously three peaks (due to three Maglev train marshalings). So, although the frame's center acceleration value is much bigger than bottom's center acceleration value (unfiltered curves and time-history), it is caused by the frame local vertical vibration and the vibration is high frequency (more than $50 \mathrm{~Hz}$ ). The different low-frequency vibration is little caused by the overall vertical vibration (less than $50 \mathrm{~Hz}$ ). Due to the China's <High-Speed Railway Design Standard $>$, the bridge's limited value of vertical acceleration at ballast less track is $0.5 \mathrm{~g}$ considering the $20-\mathrm{Hz}$ low-pass filter. The limited filter value references the European Standard. Because the vibration of frame and bottom belongs to two different frequency domains, using the filter limited value (20-Hz low-pass filter) to filter the vertical acceleration curves based on the China's $<$ High-Speed Railway Design Standard $>$ is slightly unsuitable.

\section{Conclusions}

This paper considered the PID active suspension control and used the delicate FEM model of bridge and at-ground structure based on co-simulation of SIMPACK and ANSYS. Firstly, the Maglev train-bridge vertical system coupling vibration model was validated based on one $20-\mathrm{m}$ simply supported beam load test of a low-medium-speed maglev test line. Then, the Maglev train-at-ground structure vertical system coupling vibration model was established to be analyzed. The main conclusions are shown:

1. The Maglev train-at-ground structure vertical system coupling vibration model based on the co-simulation method is reliable.

2. Being supported by the strong uniform elastic subgrade, the first-order nature frequency of the at-ground structure is $32.9 \mathrm{~Hz}$. Due to the frame local vibration, the acceleration of frame's center is $6.017 \mathrm{~m} / \mathrm{s}^{2}$, which is bigger than the bottom's center acceleration (1.428 $\mathrm{m} / \mathrm{s}^{2}$ ). The acceleration of frame's center is much bigger than the bottom's center acceleration between 50 and $100 \mathrm{~Hz}$ and has little difference within $50 \mathrm{~Hz}$. Comparing with the vibration of bottom, the frame shows the high frequency vibration (more than $50 \mathrm{~Hz}$ ), which lead to the acceleration of frame's center is much bigger than the bottom's center in time-history. In view of the obvious difference between at-ground structure and the conventional bridge, the vibration evaluate standard should be researched in further study. 
Acknowledgements This research was supported by National Natural Science Foundation of China (NSFC, Grant Nos. 51308469, 51378429).

Open Access This article is distributed under the terms of the Creative Commons Attribution 4.0 International License (http://crea tivecommons.org/licenses/by/4.0/), which permits unrestricted use, distribution, and reproduction in any medium, provided you give appropriate credit to the original author(s) and the source, provide a link to the Creative Commons license, and indicate if changes were made.

\section{References}

1. Deng XX (2009) The study of middle-low speed maglev vehicle system dynamic. Southwest Jiaotong University, Chengdu (in Chinese)

2. Wang LD (2014) Research on key technology of low and medium speed maglev guideway girder. Southwest Jiaotong University, Chengdu (in Chinese)

3. Shi J, Yau JD, Wang YJ (2012) Dynamic response of guideway girders due to high-speed maglev trains moving at resonant speeds. Eng Mech 29(12):196-203 (in Chinese)

4. Yau JD (2009) Vibration control of maglev vehicles traveling over a flexible guideway. J Sound Vib 321:184-200

5. Zhao CF, Zhai WM (2002) Maglev vehicle/guideway vertical random response and ride quality. Veh Syst Dyn 38(3):185-210

6. Cai CB (2001) Maglev vehicle/elevated-beam guideway vertical coupling dynamics. J China Railw Soc 23(5):27-33 (in Chinese)
7. Shi J, Wei QC, Wu FY (2003) Study on vibration of the beam of magnetic levitation express railway and its control. China Saf Sci J 10:76-80 (in Chinese)

8. Jiang WL, Gao MM (2004) Study of the effect of track beam parameters on vertical coupled dynamic response of maglev vehicle-viaduct. China Railw Sci 25(3):71-75 (in Chinese)

9. Lee JS, Kwon SD, Kim MY et al (2009) A parametric study on the dynamics of urban transit maglev vehicle running on flexible guideway bridges. J Sound Vib 328(3):301-317

10. Zhai WM, Zhao CF (2005) Dynamics of maglev vehicle/guideway systems (I) - magnet/rail interaction and system stability. Chin J Mech Eng 41(7):1-10 (in Chinese)

11. Zhai WM, Zhao CF (2005) Dynamics of maglev vehicle/guideway systems (II) - modeling and simulation. Chin J Mech Eng 41(8):163-175 (in Chinese)

12. Shan CS (2014) Study on vertical coupling vibration of lowmedium speed maglev train-bridge system. Southwest Jiaotong University, Chengdu (in Chinese)

13. Yau JD (2009) Aerodynamic vibrations of a maglev vehicle running on flexible guideways under oncoming wind actions. J Sound Vib 329:1743-1759

14. Kwon SD, Lee JS, Moon JW et al (2008) Dynamic interaction analysis of urban transit maglev vehicle and guideway suspension bridge subjected to gusty wind. Eng Struct 30:3445-3456

15. Shi J, Wei QC, Zhao Y (2007) Analysis of dynamic response of the high-speed EMS maglev vehicle/guideway coupling system with random irregularity. Veh Syst Dyn 45(12):1077-1095

16. Liang $X$ (2015) Study on maglev vehicle/guideway coupled vibration and experiment on test rig for a levitation stock. Southwest Jiaotong University, Chengdu (in Chinese) 